\title{
'Spontaneous' rupture of the diaphragm
}

\author{
G.A.J. McIndoe and N.F.G. Hopkins
}

General Hospital, Northampton NN1 5BD, UK.

\begin{abstract}
Summary: A case of 'spontaneous' rupture of the diaphragm with herniation and strangulation of the stomach is reported. The diagnosis was made by endoscopy and repair achieved via laparotomy. The patient has made a full recovery.
\end{abstract}

\section{Introduction}

'Spontaneous' is the term used to describe rupture of the diaphragm occurring as a result of the development of an excessive pressure gradient between the abdomen and thorax, as opposed to rupture caused by direct trauma.

The condition is rare and dangerous but a low index of clinical suspicion may make it difficult to recognize. We report a case that illustrates the condition and its complications. The diagnosis and subsequent management are described.

\section{Case report}

A 23 year old labourer presented with a 24 hour history of epigastric pain radiating to the left side of the chest and back. The pain had come on suddenly whilst the patient was digging vigorously and had been followed by nausea and later vomiting. He had previously had mild epigastric pain and a chest infection, and was in the habit of smoking 30 cigarettes and drinking 5 to 10 pints of beer per day.

On examination, he appeared ill and was reluctant to breathe deeply. He had a tachycardia but was not shocked. Breath sounds were reduced at the left lung base and he was tender in the epigastrium. He was later observed to vomit altered blood. Chest X-ray showed shadowing at the left base but no effusion. Abdominal films were unremarkable. Blood tests were normal except for a leucocytosis of $21.9 \times 10^{9} / 1$.

A diagnosis of haematemesis and left lower lobe pneumonia was made and he was admitted to hospital for observation and a course of antibiotics and physiotherapy. Upon making a partial recovery, the patient took his own discharge only to be re-admitted later in a state of shock. Examination of his abdomen remained unremarkable but chest X-ray showed fur-

Correspondence: N.F.G. Hopkins, F.R.C.S.

Accepted: 17 December 1985 ther left lower lobe collapse and the development of a pleural effusion (Figure 1). His leucocytosis had increased to $31.2 \times 10^{9} / 1$ and his urea had risen from normal levels to $25.3 \mathrm{mmol} / 1$.

Deterioration in his condition required his transfer to the Intensive Care Unit and intermittent positive pressure ventilation. Eight hundred $\mathrm{ml}$ of bloodstained fluid was aspirated from his left pleural cavity. He continued to be treated on the basis of an atypical pneumonia but his condition did not improve and he required continued ventilation and a tracheostomy. Seventeen days later his chest X-ray showed an air fluid level in the left lower zone (Figure 2). A drain was inserted which drained foul-smelling fluid. This was noted to resemble the fluid draining from his nasogastric tube and the volumes drained from the two tubes had an inverse relationship. Gastroscopy was therefore performed, demonstrating a normal oeso-

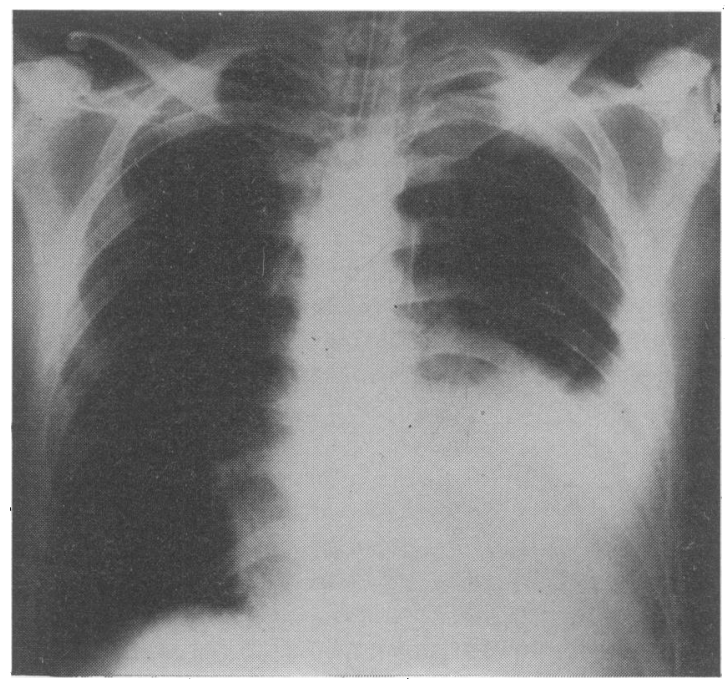

Figure 1 Chest X-ray showing left pleural effusion.

The Fellowship of Postgraduate Medicine, 1986 


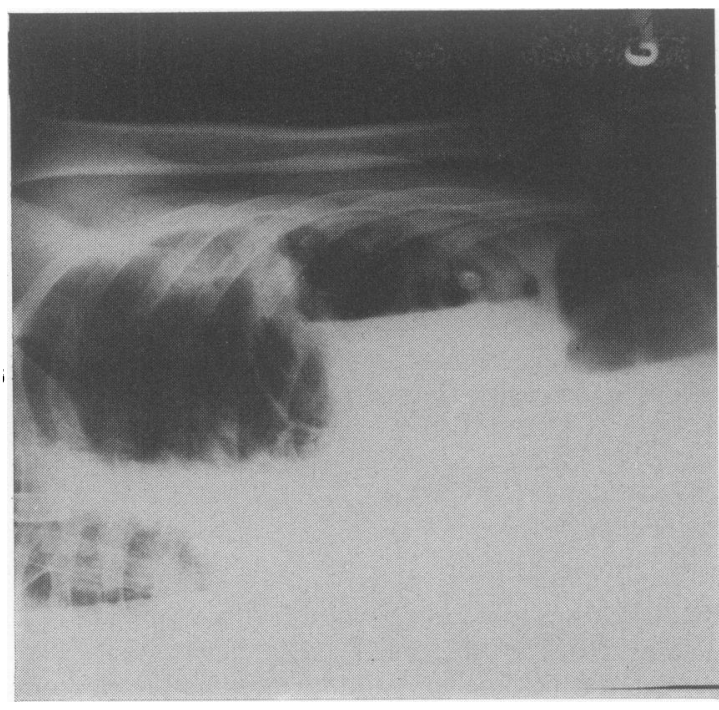

Figure 2 Chest X-ray taken in lateral, decubitus showing air-fluid level above the left hemidiaphragm.

phagus and proximal stomach, but it was not possible to enter the gastric antrum. Air insufflated via the gastroscope escaped via the chest drain making the diagnosis of gastro-pleural fistula.

Laparotomy was performed which revealed a stellate rupture of the dome of the left hemidiaphragm. Much of the greater omentum and the anterior wall of the stomach had herniated into the chest and become strangulated. The defect in the diaphragm was enlarged slightly to reduce the necrotic, foul-smelling stomach and omentum back into the abdomen. All necrotic tissues were excised and the stomach reconstructed as for a Billroth I gastrectomy. The cavity in the left chest was irrigated via the diaphragmatic defect, a chest drain placed in the cavity and the diaphragm repaired using Polydioxanone (PDS) sutures.

Post-operatively the patient's condition improved rapidly and with no further complications. The empyema cavity in his chest closed around the drain as demonstrated by repeat sinograms. Formal decortication was not required.

\section{Discussion}

Rupture of the diaphragm occurs as a result of penetrating and crush injuries but may also occur 'spontaneously'. This last category is very rare but has been reported following heavy physical effort (Salaman et al., 1969), sudden twisting movements (Bekassy et al., 1973), childbirth (Dave et al., 1973; Kumar \& Rao, 1982) and coughing fits (Dutta, 1975).

Both 'spontaneous' rupture of the diaphragm and that resulting from blunt trauma are probably caused by sudden increase in abdominal pressure resulting in a pressure gradient across the diaphragm. The left hemi-diaphragm is much more commonly affected than the right because the liver protects the right side and because the left hemi-diaphragm is weaker (Bekassy et al., 1973). All reported cases of 'spontaneous' rupture are left sided.

The most dangerous complication of ruptured diaphragm is herniation of abdominal contents into the left hemithorax and subsequent strangulation. The stomach, greater omentum and colon are most particularly at risk (Bekassy et al., 1973).

The case we report is almost certainly a case of spontaneous rupture of the diaphragm resulting from the physical effort of digging. Violent vomiting may also have contributed to the diaphragmatic rupture or at least encouraged the herniation of abdominal contents into the left hemithorax, where they subsequently strangulated. The correct diagnosis was delayed as a result of a low index of clinical suspicion plus lack of patient co-operation in that he repeatedly took his own discharge from hospital. The accurate history reported here was not obtained from the patient until he had made a full recovery and was not available at the time of diagnostic dilemma.

Drainage of the pleural effusion could have damaged the herniated stomach and should not have been performed had the diagnosis been suspected. Such a complication has been previously reported (Salaman et al., 1969).

The chest X-ray in these cases is invariably abnormal but rarely diagnostic. Early films in this case suggested pneumonia and pleural effusion. Subsequently the air-filled stomach produced an appearance confused with a pneumothorax. Barium studies will usually make the diagnosis (Salaman et al., 1969; Bekassy et al., 1973; Dutta, 1975) but in this case endoscopy was employed. Diagnosis by this method has not been previously reported.

Previous reports recommend thoracotomy as the preferable surgical approach, sometimes extended to thoraco-abdominal, because reduction of the herniated bowel may be difficult. There is only one previous report of repair via laparotomy (Kumar \& Rao, 1982). In the case we report, reduction of the hernia was achieved by a small extension of the diaphragmatic rupture which was subsequently easy to repair. Assessment and resection of the damaged bowel required full abdominal exposure but the additional morbidity associated with thoracotomy was not necessary. 


\section{References}

BEKASSY, S.M., DAVE, K.S., WOOLER, G.H. \& IONESCU, M.I. (1973). 'Spontaneous' and traumatic rupture of the diaphragm. Annals of Surgery, 177, 320.

DAVE, K.S., BEKASSY, S.M., WOOLER, G.H. \& IONESCU, M.I. (1973). 'Spontaneous' rupture of the diaphragm during delivery. British Journal of Surgery, 60, 666.

DUTTA, T. (1975). Spontaneous rupture of the diaphragm due to pertussis. Journal of Pediatric Surgery, 10, 147. KUMAR, E.B. \& RAO, A.B. (1982). Spontaneous rupture of the diaphragm during delivery. West Indian Medical Journal, 31, 93.

SALAMAN, J., FELLER, N. \& LEVY, M.J. (1969). A case of spontaneous rupture of the diaphragm. Journal of Thoracic and Cardiovascular Surgery, 58, 221. 